\title{
GMR
}

\section{Comparative transcriptome analysis of self-incompatible flower stigmas and self-compatible bud stigmas following self-pollination in broccoli}

\author{
H.F. Yu ${ }^{1}$, J.S. Wang ${ }^{1}$, X.G. Sheng ${ }^{1}$, Z.Q. Zhao ${ }^{1}$, Z.R. Qi ${ }^{2}$ and H.H. Gu ${ }^{1}$ \\ ${ }^{1}$ Institute of Vegetables, Zhejiang Academy of Agricultural Sciences, \\ Hangzhou, Zhejiang, China \\ ${ }^{2}$ Agriculture Technology Promotion Center, Cixi, Zhejiang, China \\ Corresponding author: H.H. Gu \\ E-mail: guhh@mail.zaas.ac.cn \\ Genet. Mol. Res. 16 (1): gmr16019018 \\ Received July 21, 2016 \\ Accepted February 17, 2017 \\ Published March 30, 2017 \\ DOI http://dx.doi.org/10.4238/gmr16019018
}

Copyright $\left({ }^{\circ} 2017\right.$ The Authors. This is an open-access article distributed under the terms of the Creative Commons Attribution ShareAlike (CC BY-SA) 4.0 License.

\begin{abstract}
DH07 is a DH line of Class I $S$-haplotype in broccoli (Brassica oleracea var. italica), in which stigmas of flowers show self-incompatibility (SI) and stigmas of buds show self-compatibility (SC). The molecular mechanisms that lead to stigmas at different developmental stages having different responses to self-pollination are yet unknown. In the present study, comparative transcriptome profiling of the stigmas of flowers and buds before and after self-pollination was performed by RNA-sequencing using an Illumina HiSeq ${ }^{\mathrm{TM}}$ 2000. A total of $80,102,897$ reads were generated for further analysis in four libraries. Comparisons of the transcriptome profiles before and after self-pollination revealed 579 differentially expressed genes (DEGs) in the stigmas of buds (SBs); of these, 431 DEGs showed increased and 148 DEGs showed decreased transcript abundance after self-pollination in SBs. There were a total of 686 DEGs between unpollinated stigmas of flowers (SFs) and pollinated SFs, among which, 517 DEGs were
\end{abstract}

Genetics and Molecular Research 16 (1): gmr16019018 
up regulated and 169 DEGs were down regulated. Following the selfpollination, 379 identified DEGs were common in both SBs and SFs. It was found that ARR7-like and oxysterol-binding family protein related DEGs could play key roles in SI or SC signal transduction. The results obtained in this study would form the foundation for further studies on investigating the molecular mechanisms of SI and SC in Brassica.

Key words: RNA-sequence; Self-incompatibility; Stigma; Broccoli; Brassica oleracea var. italic

\section{INTRODUCTION}

Self-incompatibility (SI) is a reproductive barrier; it is a genetic mechanism through which flowering plants prevent self-pollination to ensure outcrossing and genetic diversity. In Brassicaeae, SI is sporophytically controlled by three $S$-locus genes: $S L G(S$-locus glycoprotein gene), $S R K$ ( $S$-locus receptor kinase gene), and $S C R$ ( $S$-locus cysteine-rich protein gene) (Stein et al., 1991; Schopfer et al., 1999). SRK is pistil determinant and SCR is pollen $S$-determinant in Brassica. SLG mediates pollen adhesion (Kachroo et al., 2001). SLG, SRK, and SCR are linked closely as a Mendelian genetic locus. When a compatible pollen grain lands on stigma, it swells and a pollen tube grows, whereas when self-pollen attaches to the stigma, SCR binds specifically to SRK of the same $S$-haplotype (Takayama et al., 2001), which triggers an SI signal pathway to reject the self-pollen.

Besides SCR, SRK, and SLG, there are other components also that participate in the SI signal pathway, such as MLPK (M-locus protein kinase; Kakita et al., 2007), ARC1 (ARM repeat containing 1; Stone et al., 1999), and EXO70A1 (Samuel et al., 2009). MLPK, a membrane-anchored cytoplasmic protein kinase (Kakita et al., 2007), interacts directly with SRK to phosphorylate ARC1, which is a U-box protein with E3 ubiquitin ligase activity (Stone et al., 2003). Thereafter, the phosphorylated ARC1 mediates degradation of Exo70A1, which leads to the final rejection of the self-pollen (Samuel et al., 2009). However, ARC1 and Exo70A1 represent only one branch of the SI signaling network (Stone et al., 2003; Samuel et al., 2009; Tantikanjana et al., 2010). Thus, the SI response is more complex (Kitashiba et al., 2011).

For producing hybrid seeds of cabbage, cauliflower, broccoli, Brussels sprout, and kale, the SI character is utilized (King, 2003). Breeding of plants having SI could be achieved under high levels of humidity or by bud pollination. Some young buds are opened 3-4 days before flowering and pollen from an open flower on the same plant are transferred to the stigmas of the buds. When pollinated with the pollens of the same plant, stigmas of flowers (SFs) show SI, and stigmas of buds (SBs) show self-compatibility (SC). SI is based on self/nonself-pollen-pistil recognition and subsequent inhibition of hydration, germination, or pollen tube growth of the self-pollen. However, it appears that there are no recognition interactions between pollens and pistils of buds. There is no study reporting the molecular mechanisms underlying the differences of seed setting ability of pistils at different developmental stages after self-pollination in broccoli. The study on the molecular mechanisms of the stigmas, at different developmental stages, responding to self-pollination is of fundamental importance in broccoli biology.

By quantifying differences in the abundance of the expression of genes through the analysis of transcriptome prior to, and after pollination of SFs and SBs, we can identify the

Genetics and Molecular Research 16 (1): gmr16019018 
subset of genes whose abundance is modified following SI/SC response. These analyses could probably reveal genes triggered by self-pollen on the stigma and would identify the genes required for promoting SI and SC responses, respectively.

\section{MATERIAL AND METHODS}

\section{Plant materials}

DH07 is a DH line of Class I $S$-haplotype SI in broccoli (Brassica oleracea var. italica) (Yu et al., 2014), in which SFs show SI and SBs show SC as revealed by self-compatibility index (SCI). SCI is calculated as the ratio of percentages between self-pollinated seed number and self-pollinated pistils. In this study, SCI was determined by dividing the number of seeds by the number of pistils. Plants with an SCI $<1$ in broccoli are considered to have SI, whereas higher values indicate that the plant has SC. Field experiments were performed at Yangdu Experimental Station of Zhejiang Academy of Agricultural Sciences. Broccoli seeds were sown in potted tray in August 2012 and the seedlings were transplanted to a greenhouse after 1 month and grown under conventional field management. From March to April 2013, the open flowers and siliques were removed, and inflorescences at bud stage were collected. After 3 days, self-pollination was performed manually on SFPs and SBPs on the same inflorescences.

The stigmas were randomly sampled at anthesis from approximately 150 fresh pistils at $0.5 \mathrm{~h}$ after being pollinated and from buds, 3-4 days before flowering. The stigmas that were not pollinated with self-pollen were cut off and collected as control at anthesis and from buds, 3-4 days before flowering. Four samples were collected and immediately put in liquid nitrogen, and stored at $-80^{\circ} \mathrm{C}$ for later use. The fresh weight of pistils of each group (pollinated and control) amounted to about $100 \mathrm{mg}$.

\section{RNA extraction and Solexa/Illumina sequencing}

The extraction of total RNA and mRNA for sequencing was performed by the Biomarker Biotechnology Corporation (Beijing, China). After the extraction of total RNA, mRNA was purified and cleaved into short fragments. Using these cleaved mRNA fragments as templates, the double-stranded cDNA was synthesized and purified according to the protocols (Liu et al., 2012). This was followed by end-repairing using T4 DNA polymerase (NEB, China), the Klenow fragment (NEB), and T4 polynucleotide kinase (NEB). At the 3 '-ends of the repaired cDNA fragments, an "A" base was inserted and the sequencing adapters were ligated to the cDNA fragments. To select templates within a desired size range, the products of the ligation reaction were separated on a $2 \%$ agarose gel. To selectively enrich the cDNA fragments, PCR amplification was performed. Four libraries were sequenced using an Illumina $\mathrm{HiSeq}^{\mathrm{TM}} 2000$.

\section{Sequence data analysis and assembly}

The fastq format raw reads were deposited in NCBI database (GenBank accession No. SRP062687). After cleaning of the raw reads by removal of the adapter sequences and reads with more than $10 \% \mathrm{Q}$, less than 20 bases, and those that were with ambiguous bases "N", the quality reads were assembled into unigenes de novo with Trinity (http://trinityrnaseq. sourceforge.net) using the parameters "K-mer $=25$, group pairs distance $=300$ " (Grabherr et

Genetics and Molecular Research 16 (1): gmr16019018 
al., 2011). Trinity software assembled short reads into longer contigs based on their overlap regions. Using their paired-end information, different contigs were assembled into the sequence of the transcripts. The assembled sequences were then compared pairwise by BLAT tool (The BLAST-Like Alignment Tool, http://genome.ucsc.edu/cgi-bin/hgBlat; Kent, 2002). Clustering analysis was performed for the samples. Ultimately, a unigene database was obtained.

\section{Analysis and screening of differentially expressed genes (DEGs)}

The number of unigenes expressed was computed using RPKM (Reads Per kb per Million reads). IDEG6 (http://telethon.bio.unipd.it/bioinfo/IDEG6/) was used to identify DEGs between the samples. The P value corresponded to the DEGs tested. The FDR (False Discovery Rate) was used to correct the P value in multiple tests and analyses by manipulating the FDR value. FDR $\leq 0.001$ and the absolute value of $\mid \log 2$ Ratio $\mid \geq 1$ were used as thresholds to determine the significance of the differences in transcript abundance.

\section{Gene annotation}

The assembled unigenes were annotated by aligning in diverse protein and nucleotide databases, including SwissProt, PIR (Protein Information Resource), PRF (Protein Research Foundation), PDB (Protein DataBank), National Center for Biotechnology Information (NCBI) non-redundant protein $(\mathrm{Nr})$ database, NCBI non-redundant nucleotide sequence $(\mathrm{Nt})$ database, Kyoto Encyclopedia of Genes and Genomes (KEGG), and Cluster of Orthologous Groups of proteins $(\mathrm{COG})$. The best annotation was selected from the matches with an E-value less than $10^{-5}$. To annotate the DEGs, the information was extracted from unigenes annotation information, and Gene Ontology (GO) and KEGG pathway enrichment of the DEGs were analyzed.

\section{Real-time quantitative RT-PCR (qRT-PCR) verification}

Real-time quantitative RT-PCR (qRT-PCR) was used to verify the digital gene expression (DGE) results. The RNA samples used for the qRT-PCR assays were the same as used for RNA sequencing. Total RNA was extracted according to the protocol accompanying the RNA extraction kit (TianGen Biotech Co. LTD, Beijing). The RNA samples were then reverse-transcribed using FastQuant RT Kit (with gDNase; TianGen, Beijing, China). Genespecific primers were designed according to the reference unigene sequences using the Primer Premier 5.0 and were synthesized by Shanghai Invitrogen Biotechnology Co. Ltd. The selected genes were verified using Applied Biosystems StepOnePlus Real-Time PCR system with a cycling temperature of $62^{\circ} \mathrm{C}$, by a single peak on the melting curve to ensure a single product. For each sample, two independent biological replicates and three technical replicates per biological replicate were tested to ensure reproducibility and reliability. The actin gene was used as an internal control for normalization of gene expression.

\section{RESULTS}

\section{Differences in the self-compatibility index of the pistils of flowers and buds}

The differences in the self-compatibility index between flower pistils (FPs) and bud 
pistils (BPs) of DH07 are shown in Figure 1C. Small and short pods, which had no or few seeds, developed from FPs after self-pollination (left in Figure 1A, B). Big and long pods with an average of $\sim 5.7$ seeds per pod developed from BPs after self-pollination (right in Figure 1A, B). The number of seeds per pistil tested indicated that FPs were self-incompatible, whereas BPs were self-compatible.

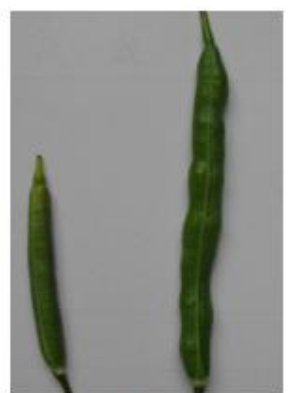

A

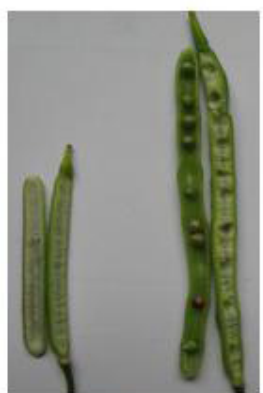

B

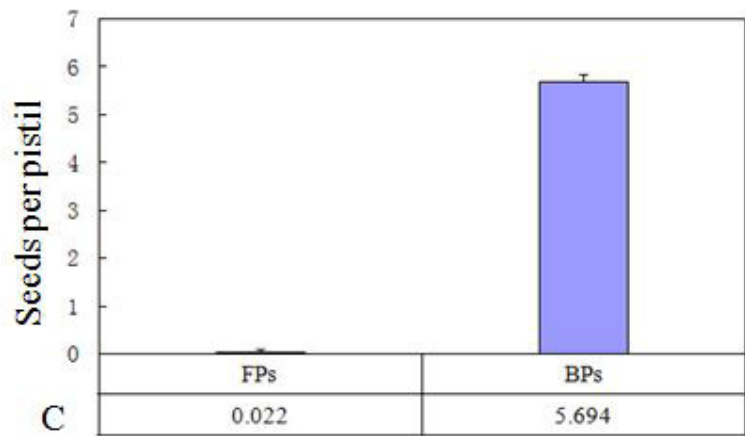

Figure 1. Seed setting of flowers pistils (FPs) (left in A and B) and buds pistils (BPs) after self-pollination (right in A and B). C. Number of seeds per pistil after self-pollination, i.e. self-compatibility index.

\section{Sequence analysis and assembly}

To obtain a global overview of the transcriptome and differences in the expression of genes in SFs and SBs following self-pollination, four cDNA samples from pollinated and unpollinated SFs and SBs were prepared and sequenced using an Illumina HiSeq ${ }^{\mathrm{TM}}$ 2000. After stringent quality assessment and data screening, 80,102,897 reads with average 97.57\% Q20 bases were selected as high quality reads for further analysis in the four libraries. Overall, $20,317,216$ and $20,139,046$ reads were sequenced in the libraries prepared from the unpollinated SFs (USFs) and pollinated SBs (PSBs), respectively. There were 19,815,945 and $19,830,690$ reads in the libraries prepared from the un-pollinated SBs (USBs) and of pollinated SBs (PSBs), respectively. After the sequence data analysis and assembly, short-read sequences were assembled into 65,915 unigenes. There were 10,036 unigenes that were longer than $1 \mathrm{~kb}$ and 5,927 unigenes that were longer than $2 \mathrm{~kb}$.

\section{Identification of differentially expressed genes in stigmas of different developmental stages following self-pollination}

To identify clusters with functional enrichment, hierarchical clustering was performed based on gene expression patterns (Figure 2). The gene expression in SBs showed fewer differentially expressed genes (DEGs) after self-pollination; in contrast, there were more DEGs in SBs after self-pollination. In SBs, 579 DEGs were identified (Table S1), of which 431 DEGs showed increased and 148 DEGs showed decreased transcript abundance after self-pollination. There were a total of 686 DEGs between un-pollinated SFs and pollinated SFs (Table S2). Among them, 517 DEGs were up-regulated and 169 were down-regulated.

Following self-pollination, 379 DEGs that were identified were common in both SBs and SFs (Table S3). Among these, 26 DEGs showed decreased and 351 DEGs showed 
increased transcript abundance after self-pollination, and another 2 DEGs had different expression change in both SBs and SFs. Cytoskeleton, signal transduction, posttranslational modification, and transcription genes were found among the down-regulated DEGs that were common to the stigmas at both the developmental stages after self-pollination.

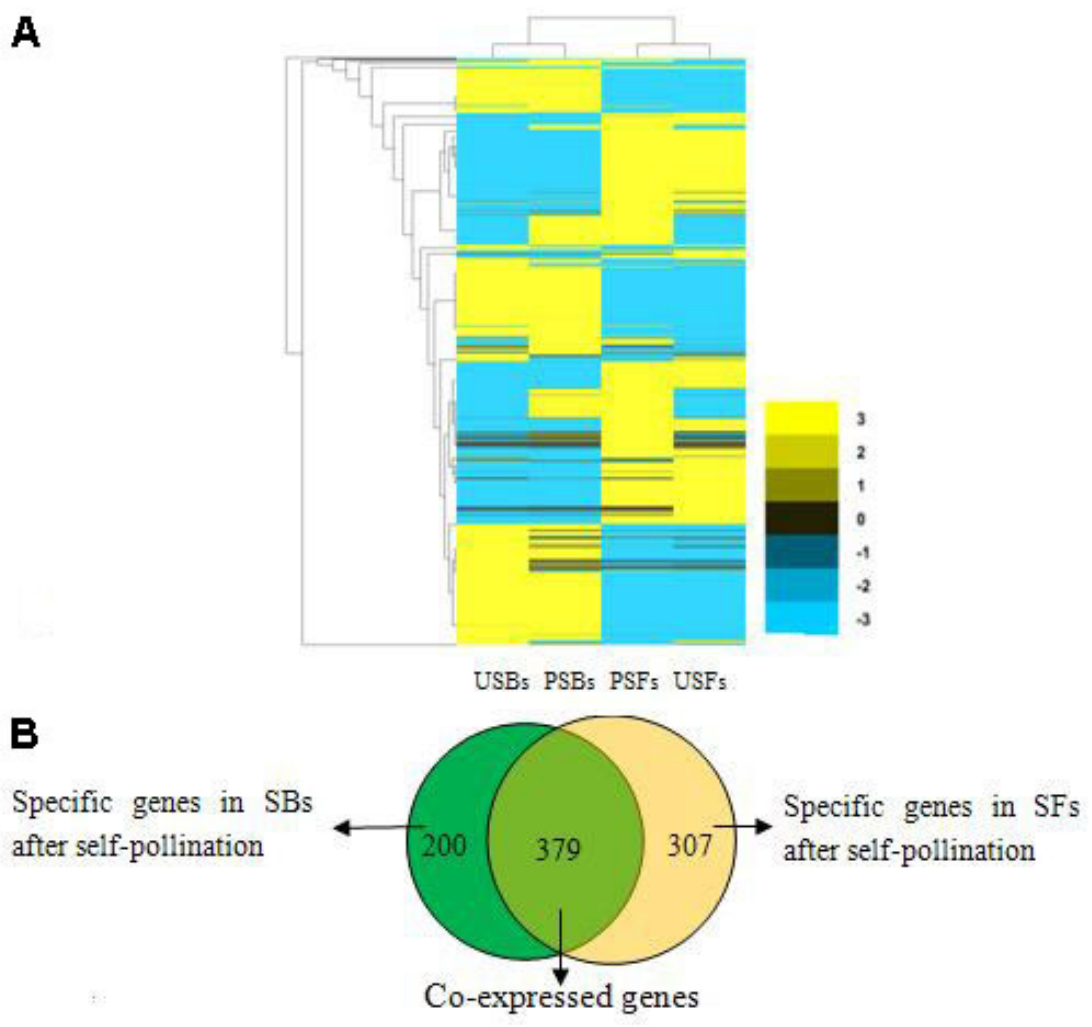

Figure 2. Differentially expressed genes between stigmas of buds (SBs) and stigmas of flowers (SFs) before and after self-pollination. A. Hierarchical cluster analysis of differentially expressed genes based on the log ratio fold change data. The column and row indicate the sample and gene, respectively; the color scale indicates the gene expression level: yellow color represents increased transcript abundance and blue color represents decreased transcript abundance. USBs and PSBs represent un-pollinated and pollinated SBs, respectively; USFs and PSFs represent un-pollinated and pollinated SFs, respectively. B. Venn diagram displays the number of specific genes in SFs and SBs after self-pollination and co-expressed genes.

\section{Functional classification of genes induced by self-pollination using GO and KEGG analysis}

The GO assignment system can assist in understanding the characteristics of gene function at a macroscopic level. It was used to acquire functional information regarding the DEGs. Using GO analysis, all the DEGs were assigned into three categories: cellular component, molecular function, and biological process. In the comparison of SBs, "cell" (GO: 0005618), "cell part" (GO: 0044464), and "cellular process" (GO: 0009987) were the three 
most highly enriched terms. The same most highly enriched terms, as in the comparison of SBs, were present in the comparison of SFs. However, the total number of GO terms was more in the comparison of SBs than in the comparison of SFs.

KEGG analysis could further the research on the biological functions of gene complexes. KEGG analysis showed that 37 enriched KEGG pathways were common in the comparisons of SBs and SFs. The DEGs common in both SBs and SFs were mainly for the photosynthesis antenna proteins, RNA polymerase, those involved in circadian rhythm of plant, starch and sucrose metabolism, plant hormone signal transduction, pyrimidine metabolism, and purine metabolism. "Glycine, serine and threonine metabolism" (ko00260), "beta-Alanine metabolism" (ko00410), and "Fatty acid metabolism" (ko00071) were significantly enriched in the DEGs of SBs (Table 1). For the DEGs of SFs, "Protein processing in endoplasmic reticulum" (ko04141), "Endocytosis" (ko04144), "Carotenoid biosynthesis" (ko00906), and "Porphyrin and chlorophyll metabolism" (ko00860) were significantly enriched.

Table 1. Significantly enriched KEGG pathways in the RNA-Seq analysis.

\begin{tabular}{l|c|c|c}
\hline Term & Sample number & Background number & Comparison \\
\hline Glycine, serine and threonine metabolism & 4 & 83 & SBs \\
\hline beta-Alanine metabolism & 3 & 57 & SBs \\
\hline Fatty acid metabolism & 3 & 89 & SBs \\
\hline Protein processing in endoplasmic reticulum & 13 & 280 & SFs \\
\hline Endocytosis & 5 & 165 & SFs \\
\hline Carotenoid biosynthesis & 3 & 55 & SFs \\
\hline Porphyrin and chlorophyll metabolism & 3 & 82 & SFs \\
\hline
\end{tabular}

\section{Validation of RNA-Seq-based gene expression}

To validate the DEGs identified in the RNA-sequencing analysis, 10 genes with altered expression in SBs or SFs, following pollination, were randomly selected for qRTPCR (Table S4). The expression patterns from qRT-PCR were compared with the results of the RNA-Sequencing expression analysis. The results showed that all the 10 genes had the same expression patterns in the qRT-PCR analysis as in the RNA-Sequencing analysis, which proves the reliability and accuracy of the RNA-Sequencing expression analysis (Figure 3).

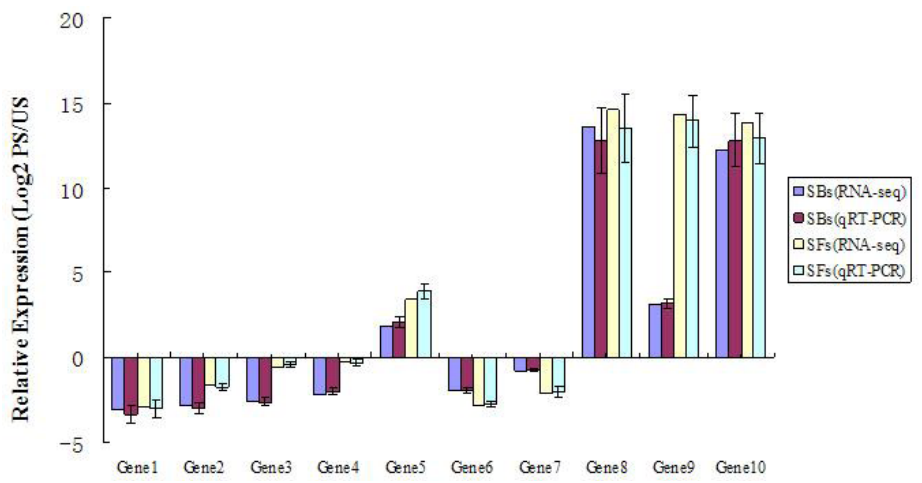

Figure 3. Validation of the expression levels of DEGs obtained by RNA-Seq by qRT-PCR. Gene1, gene2, gene3, gene4, gene5, gene6, gene7, gene8, gene9, and gene 10 denote T2_Unigene_BMK.21180, T1_Unigene_BMK.6827, T1 Unigene_BMK.10969, T4_Unigene_BMK.25402, T4_Unigene_BMK.19074, T4_Unigene_BMK.30688, T2_Unigene BMK.26534, T3_Unigene_BMK.9073, T4_Unigene_BMK.17221, and T4_Unigene_BMK.15905, respectively.

Genetics and Molecular Research 16 (1): gmr16019018 


\section{Expression analysis of known genes related SI in RNA-Seq}

The expression levels of $S R K, S L G, A R C 1$, and EXO70A1 in SBs were far lower in SFs; especially SLG had a very high expression level in SFs (Figure 4). The expression levels of SRK, ARC1,EXO70A1-like, THL in the pollinated SBs and SFs were not different obviously from in the unpollinated SBs and SFs, so pollination seemed no effect on the expression of these four genes in SBs and SFs. THL had higher expression level in SBs than in SFs. The expression level of THL in USBs and PSBs was almost the same, indicating that selfpollination mostly had no effect on the expression of $T H L$ in SBs. The expression of $T H L$ in SFs was not only low, but also decreased by nearly half after self-pollination. The expression of aquaporin was more abundant in SFs than in SBs. Pollination could promote the expression of aquaporin in both SFs and SBs.

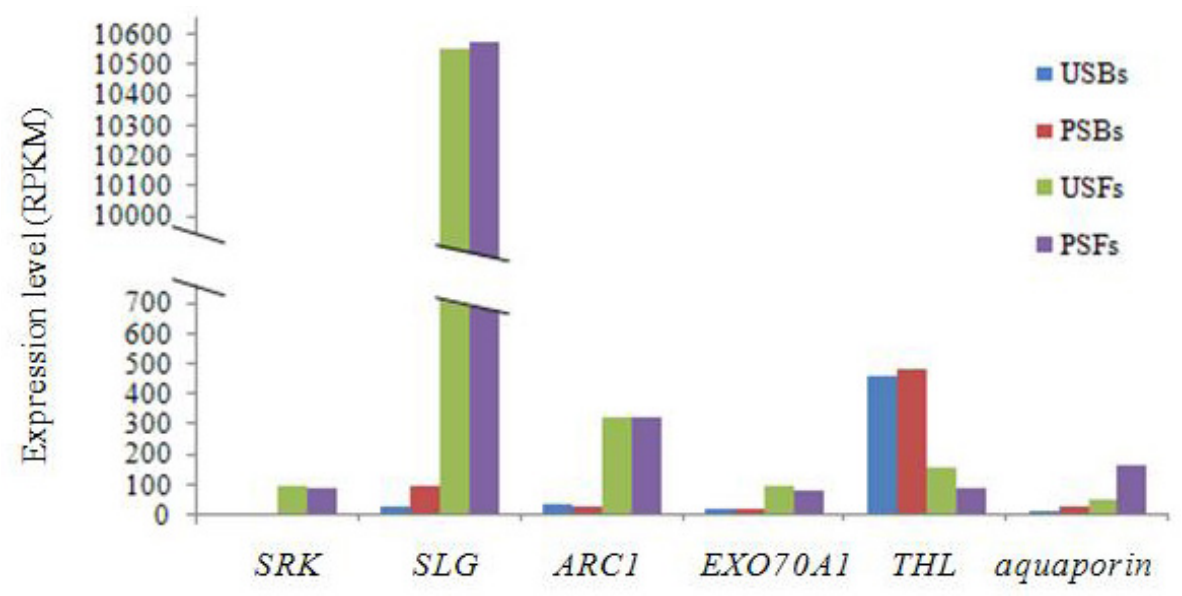

Figure 4. Expression levels of known genes related to SI in USBs (Un-pollinated Stigmas of Buds), PSBs (Pollinated Stigmas of Buds), USFs (Un-pollinated Stigmas of Flowers), and PSBs (Pollinated Stigmas of Flowers) as determined by RNA-seq.

\section{DISCUSSION}

In this study, the transcriptomes of the stigmas in buds and flowers, the two different development stages, following self-pollination in broccoli DH07 were evaluated using RNASeq analysis. In DH07, the stigmas of flowers showed SI and stigmas of buds showed SC. Although there are many studies reporting the results of molecular research on SI in Brassica, the molecular mechanism of different developmental stigmas in response to self-pollination remains poorly understood. In this RNA-Seq analysis, 80 million raw reads with an average of 97.57\% Q20 bases were generated; 579 DEGs in SBs and 686 DEGs in SFs were identified. These results suggested that more genes could be involved in self-pollination regulatory network in the stigmas of flowers than in the stigmas of buds. KEGG and functional enrichment analysis revealed that most of the differential genes were annotated to be involved protein processing in endoplasmic reticulum, endocytosis, porphyrin, and chlorophyll metabolism etc.

$S R K$ was expressed specially in stigmas and was the pistil $S$-determinant in SI plants, which had the highest expression levels on the day of anthesis and remained highly expressed in

Genetics and Molecular Research 16 (1): gmr16019018 
the early stages after self-pollination (Liu et al., 2007). Without incompatible pollen on stigma, THL was able to bind the SRK kinase domains, and inhibited the SRK activity (Bower et al., 1996; Cabrillac et al., 2001). When incompatible pollen landed on stigma, SRK interacted specifically with SCR, and was released from THL and activated. It was then directed with ARC1 to re-localize to the proteasomes, where ARC1 targets EXO70A1 (Kachroo et al., 2001; Takayama et al., 2001; Samuel et al., 2008; Wang et al., 2014). A complex SI signal pathway was activated in the stigma, which ultimately led to rejection of self-pollen. In this study, the expression levels of SRK, SLG, ARC1, and EXO70A1 in SBs were far lower in SFs, and selfpollination had little to no effect on the expression levels of the genes in both SBs and SFs. However, THL had higher expression level in SBs than in SFs. Self-pollination had almost no effect on the expression of THL in SBs. The expression of THL in SFs was not only low, but also reduced to nearly half, after self-pollination. The results of this study revealed that $T H L$ had inhibitory effect in SI reaction of broccoli, and that stigmas of buds were self-compatible, which might be related to the high expression of THL and low expression of SRK, SLG, and $A R C 1$. Exo70A1 was a potential substrate for $\mathrm{ARC1}$ and was required for compatible pollenstigma interactions (Samuel et al., 2008). However, in this study, the expression levels of EXO70A1 in SBs were far lower in SFs and pollination had little to no effect on its expression levels both in SBs and SFs, which indicates that EXO70A1 could not be the negative regulator of SI. Aquaporin was reported to be required for the Brassica self-incompatibility response (Ikeda et al., 1997). However, this study could not confirm the relationship of aquaporin and self-incompatibility.

There were 2 DEGs T3_Unigene_BMK.15656 and T4_Unigene_BMK.17065, which had different expression change in both $\overline{\mathrm{SB}}$ s and SFs. This shows that after self-pollination, the expression levels of both T3_Unigene_BMK.15656 and T4_Unigene_BMK.17065 were reduced in SBs, and were increased in SFs. The expression levels of T3_Unigene_BMK.15656 and T4 Unigene BMK.17065 are highly similar with those of Brassica napus two-component response regulator ARR7-like and Arabidopsis lyrata oxysterol-binding family protein. The relationship between these two DEGs and SI or SC is unknown and needs further study.

Previous studies revealed that the adherence of compatible pollen grain to the stigmatic papillae results in hydraulic connectivity with the stigma, because of which pollen grains germinate and produce tubes that penetrate the stigmatic cell walls and traverse the pistil down to the ovules, where fertilization takes place (Swanson et al., 2004). This report showed that two DEGs encoding KDEL-tailed cysteine endopeptidase CEP1 and pollen-specific leucinerich repeat extensin-like protein 4 had up-regulated expression in SBs after self-pollination, and had no change in expression levels in SFs, which indicated that KDEL-tailed cysteine endopeptidase CEP1 and pollen-specific leucine-rich repeat extensin-like protein 4 are possibly involved in the SC response.

\section{Conflicts of interest}

The authors declare no conflict of interest.

\section{ACKNOWLEDGMENTS}

The authors acknowledge the support obtained from the Office of Science and Technology of Zhejiang Province for Key Agriculture Development Projects (Foundation item

Genetics and Molecular Research 16 (1): gmr16019018 
\#2016C02051-5-1) and Agricultural Science and Technology Cooperation Projects between Taizhou People's Government and Zhejiang Academy of Agricultural Sciences (\#TYD001-2 and \#TYD-005), as well as from the Science and Technology Department of Ningbo (\#2015C110008) and Zhejiang Academy of Agricultural Sciences (\#2015CX06).

\section{REFERENCES}

Bower MS, Matias DD, Fernandes-Carvalho E, Mazzurco M, et al. (1996). Two members of the thioredoxin-h family interact with the kinase domain of a Brassica S locus receptor kinase. Plant Cell 8: 1641-1650.

Cabrillac D, Cock JM, Dumas C and Gaude T (2001). The $S$-locus receptor kinase is inhibited by thioredoxins and activated by pollen coat proteins. Nature 410: 220-223. http://dx.doi.org/10.1038/35065626

Grabherr MG, Haas BJ, Yassour M, Levin JZ, et al. (2011). Full-length transcriptome assembly from RNA-Seq data without a reference genome. Nat. Biotechnol. 29: 644-652. http://dx.doi.org/10.1038/nbt.1883

Ikeda S, Nasrallah JB, Dixit R, Preiss S, et al. (1997). An aquaporin-like gene required for the Brassica self-incompatibility response. Science 276: 1564-1566. http://dx.doi.org/10.1126/science.276.5318.1564

Kachroo A, Schopfer CR, Nasrallah ME and Nasrallah JB (2001). Allele-specific receptor-ligand interactions in Brassica self-incompatibility. Science 293: 1824-1826. http://dx.doi.org/10.1126/science.1062509

Kakita M, Murase K, Iwano M, Matsumoto T, et al. (2007). Two distinct forms of M-locus protein kinase localize to the plasma membrane and interact directly with S-locus receptor kinase to transduce self-incompatibility signaling in Brassica rapa. Plant Cell 19: 3961-3973. http://dx.doi.org/10.1105/tpc.106.049999

Kent WJ (2002). BLAT--the BLAST-like alignment tool. Genome Res. 12: 656-664. http://dx.doi.org/10.1101/gr.229202

King GJ (2003). Using molecular allelic variation to understand domestication processes and conserve diversity in Brassica crops. Acta Hortic. 598: 181-186. http://dx.doi.org/10.17660/ActaHortic.2003.598.26

Kitashiba H, Liu P, Nishio T, Nasrallah JB, et al. (2011). Functional test of Brassica self-incompatibility modifiers in Arabidopsis thaliana. Proc. Natl. Acad. Sci. USA 108: 18173-18178. http://dx.doi.org/10.1073/pnas.1115283108

Liu M, Qiao G, Jiang J, Yang H, et al. (2012). Transcriptome sequencing and de novo analysis for Ma bamboo (Dendrocalamus latiflorus Munro) using the Illumina platform. PLoS One 7: e46766. http://dx.doi.org/10.1371/ journal.pone.0046766

Liu P, Sherman-Broyles S, Nasrallah ME and Nasrallah JB (2007). A cryptic modifier causing transient self-incompatibility in Arabidopsis thaliana. Curr. Biol. 17: 734-740. http://dx.doi.org/10.1016/j.cub.2007.03.022

Samuel MA, Chong YT, Haasen KE, Aldea-Brydges MG, et al. (2009). Cellular pathways regulating responses to compatible and self-incompatible pollen in Brassica and Arabidopsis stigmas intersect at Exo70A1, a putative component of the exocyst complex. Plant Cell 21: 2655-2671. http://dx.doi.org/10.1105/tpc.109.069740

Samuel MA, Yee D, Haasen KE and Goring DR (2008). 'Self' pollen rejection through the intersection of two cellular pathways in the Brassicaceae: self-incompatibility and the compatible pollen response. In: Self-incompatibility in Flowering Plants. (Franklin-Tong VE, ed.). Springer-Verlag, Berlin, Germany, 173-191.

Schopfer CR, Nasrallah ME and Nasrallah JB (1999). The male determinant of self-incompatibility in Brassica. Science 286: 1697-1700. http://dx.doi.org/10.1126/science.286.5445.1697

Stein JC, Howlett B, Boyes DC, Nasrallah ME, et al. (1991). Molecular cloning of a putative receptor protein kinase gene encoded at the self-incompatibility locus of Brassica oleracea. Proc. Natl. Acad. Sci. USA 88: 8816-8820. http:// dx.doi.org/10.1073/pnas.88.19.8816

Stone SL, Anderson EM, Mullen RT and Goring DR (2003). ARC1 is an E3 ubiquitin ligase and promotes the ubiquitination of proteins during the rejection of self-incompatible Brassica pollen. Plant Cell 15: 885-898. http:// dx.doi.org/10.1105/tpc.009845

Stone SL, Arnoldo M and Goring DR (1999). A breakdown of Brassica self-incompatibility in ARC1 antisense transgenic plants. Science 286: 1729-1731. http://dx.doi.org/10.1126/science.286.5445.1729

Swanson R, Edlund AF and Preuss D (2004). Species specificity in pollen-pistil interactions. Annu. Rev. Genet. 38: 793818. http://dx.doi.org/10.1146/annurev.genet.38.072902.092356

Takayama S, Shimosato H, Shiba H, Funato M, et al. (2001). Direct ligand-receptor complex interaction controls Brassica self-incompatibility. Nature 413: 534-538. http://dx.doi.org/10.1038/35097104

Tantikanjana T, Nasrallah ME and Nasrallah JB (2010). Complex networks of self-incompatibility signaling in the Brassicaceae. Curr. Opin. Plant Biol. 13: 520-526. http://dx.doi.org/10.1016/j.pbi.2010.06.004

Wang L, Wang C, Ge TT, Wang JJ, et al. (2014). Expression analysis of self-incompatibility-associated genes in nonheading Chinese cabbage. Genet. Mol. Res. 13: 5025-5035. http://dx.doi.org/10.4238/2014.July.4.18

Genetics and Molecular Research 16 (1): gmr16019018 
Yu HF, Zhao ZQ, Sheng XG, Wang JS, et al. (2014). Identification of S-haplotypes in DH-lines of broccoli (Brassica oleracea L. var. italica). J. Hortic. Sci. Biotechnol. 89: 430-434. http://dx.doi.org/10.1080/14620316.2014.11513102

\section{Supplementary material}

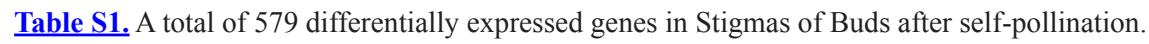

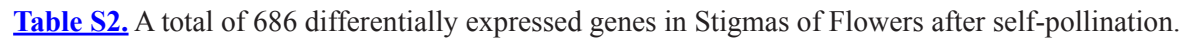

Table S3. The common 379 differentially expressed genes in BPs and FPs after self-pollination.

Table S4. qRT-PCR primer sequence.

Genetics and Molecular Research 16 (1): gmr16019018 